\title{
Propagation velocities of gas rings in collisional ring galaxies
}

\author{
E. I. Vorobyov ${ }^{1}$ and D. Bizyaev ${ }^{2}$
}

\author{
1 Institute of Physics, Stachki 194, Rostov-on-Don, Russia, and Isaac Newton Institute of Chile, Rostov-on-Don Branch \\ 2 Sternberg Astronomical Institute, Universitetsky prospect 13, Moscow, Russia; Isaac Newton Institute of Chile, \\ Moscow Branch; Department of Physics, UTEP, El Paso, Texas, USA \\ e-mail: dmbiz@sai.msu.ru
}

Received 12 August 2002 / Accepted 16 December 2002

\begin{abstract}
The propagation velocity of the first gas ring in collisional ring galaxies, i.e. the velocity at which the maximum in the radial gas density profile propagates radially in the galactic disk, is usually inferred from the radial expansion velocity of gas in the first ring. Our numerical hydrodynamics modeling of ring galaxy formation however shows that the maximum radial expansion velocity of gas in the first ring $\left(v_{\text {gas }}\right)$ is invariably below the propagation velocity of the first gas ring itself $\left(v_{\text {ring }}\right)$. Modeling of the Cartwheel galaxy indicates that the outer ring is currently propagating at $v_{\text {ring }} \approx 100 \mathrm{~km} \mathrm{~s}^{-1}$, while the maximum radial expansion velocity of gas in the outer ring is currently $v_{\text {gas }} \approx 65 \mathrm{~km} \mathrm{~s}^{-1}$. The latter value is in marginal agreement with the measurements of Higdon (1996) based on HI kinematics. Modeling of the radial $B-V / V-K$ color gradients of the Cartwheel ring galaxy also indicates that the outer ring is propagating at $v_{\mathrm{ring}} \geq 90 \mathrm{~km} \mathrm{~s}^{-1}$ for the adopted distance to the galaxy of $140 \mathrm{Mpc}$. On the other hand, the azimuthally averaged $\mathrm{H} \alpha$ surface brightness profile of the Cartwheel's outer ring does not peak exterior to those in $K$ - and $B$-bands, contrary to what would be expected for such a high propagation velocity. We show that a combined effect of $41^{\circ}$ inclination, finite thickness, and warping of the Cartwheel's disk might be responsible for the lack of angular difference in the peak positions. Indeed, the radial $\mathrm{H} \alpha$ surface brightness profiles obtained along the Cartwheel's major axis, where effects of inclination and finite thickness are minimized, do peak exterior to those at $K$ - and $B$-bands. The angular difference in peak positions implies $v_{\text {ring }}=110 \mathrm{~km} \mathrm{~s}^{-1}$, which is in agreement with the model predictions. We briefly discuss the utility of radio continuum emission and spectral line equivalent widths for determining the propagation velocity of gas rings in collisional ring galaxies.
\end{abstract}

Key words. galaxies: individual: The Cartwheel - galaxies: photometry

\section{Introduction}

Collisional ring galaxies are thought to originate in galactic collisions. A near-central axial passage of a companion galaxy through the disk of a target galaxy initiates a series of expanding ring density waves both in gas and stellar components of the target galactic disk. An expanding ring gas density wave (hereafter, gas ring) triggers high rates of massive star formation along its perimeter, if the gas density exceeds a threshold (Appleton \& Struck-Marcell 1987). The propagation velocity of gas ring, i.e. the velocity at which the maximum in radial gas density profile propagates radially in the galactic disk, is usually inferred from the radial expansion velocity of gas in the ring. Indeed, kinematical models predict that a determination of the radial expansion velocity of an individual star or HII region in the first ring gives a good indication of the ring propagation velocity (Toomre 1978). This is usually not true for higher order rings. However, rings are rather wave phenomena than material features, which implies a possible difference between

Send offprint requests to: E. I. Vorobyov, e-mail: eduard_vorobev@mail.ru the ring propagation velocity and the radial expansion velocity of particles forming the ring. Indeed, recent N-body modeling of ring galaxy formation by Athanassoula et al. (1997) have shown that the propagation velocity of the first stellar ring is usually higher than the mean velocity of stellar particles in the ring. Hence, the propagation velocity of the first gas ring may also not coincide with the radial expansion velocity of gas in the ring. If so, this would have important implications for the age estimates of collisional ring galaxies (hereafter, ring galaxies), since in many well-known systems, most notably in the Cartwheel, the rings are essentially a gas dynamical phenomenon.

In this paper we attempt to estimate the propagation velocity of gas ring from the optical and near-infrared photometry, as well as non-thermal radio emission and spectral line equivalent widths of ring galaxies. If an expanding gas ring indeed triggers high rates of star formation as predicted by Appleton \& StruckMarcell (1987), then its propagation velocity might be inferred from the properties of young stellar component formed in the ring. In Sect. 2 we explore the utility of the radial optical and near-infrared color gradients measured in the Cartwheel's disk 
(Marcum et al. 1992) for estimating the propagation velocity of the Cartwheel's outer ring. In Sect. 3 we determine the relationship between the propagation velocity of the first gas ring and the maximum radial expansion velocity of gas in the ring. The Cartwheel's radial surface brightness profiles in $K s$-band, $B$-band and $\mathrm{H} \alpha$ are compared with those predicted by a density wave model in Sect. 4. In Sect. 5 we discuss the utility of radio continuum emission and spectral line equivalent widths for determining the propagation velocity of gas rings in ring galaxies. Our main results are summarized in Sect. 6.

\section{Radial optical and near-infrared color gradients in the Cartwheel's disk}

Theory of ring galaxy formation predicts that in gas-rich galactic disks an expanding ring gas density wave might trigger a co-existing expanding wave of star formation (Appleton \& Struck-Marcell 1987). As such a density-wave-induced ring wave of star formation propagates radially from the nucleus, it leaves behind evolved stellar populations, with the youngest stars located at the current position of the wave. This might result in a radial color distribution of stars in the galactic disk, with the inner regions being redder than the outer parts of the disk. Indeed, observations of the Cartwheel ring galaxy (Marcum et al. 1992) and some other ring galaxies (Appleton \& Marston 1997) revealed the presence of the optical and nearinfrared radial color gradients in the galactic disks.

Recent attempts by Korchagin et al. (2001) and Vorobyov \& Bizyaev (2001, hereafter VB) to model the Cartwheel's radial $B-V / V-K$ color gradients using the above scenario yielded a clear success. One of the results of Korchagin et al. (2001) was to show that the model radial $B-V / V-K$ color gradients are sensitive not only to the metallicity of star-forming gas, but also to the propagation velocity of gas ring. Indeed, faster rings are expected to produce younger stellar populations provided that the rings have propagated to the same diameter in the galactic disk. This motivated us to investigate the utility of the Cartwheel's radial color gradients for estimating the ring propagation velocity.

In our previous paper (VB) we postulated the propagation velocity of the Cartwheel's outer ring as an average of all the available measurements $\left(55 \mathrm{~km} \mathrm{~s}^{-1}\right)$ and varied the metallicity of star-forming gas in the Cartwheel's disk in order to find a better agreement between the model and observed radial color gradients. In this paper we perform a more comprehensive modeling of the Cartwheel's radial $B-V / V-K$ color gradients and $Q_{B V K}$ color indices by varying the propagation velocity as well. Our intuitive expectations are that the Cartwheel's radial color gradients cannot be reproduced equally well for any value of the outer ring propagation velocity. If so, we would be able to estimate the outer ring propagation velocity from the available optical and near-infrared photometry of the Cartwheel ring galaxy. The equations and methods used to model the colors and color indices are explained in detail in VB and references therein. The population synthesis code used in this work is explained in detail in Mayya (1995, 1997). Using stellar evolutionary (Schaller et al. 1992) and atmospheric (Kurucz 1992) models, this code synthesizes a number of observable quantities in the optical and near-infrared passbands, which are suitable for comparison with the observed properties of giant star-forming complexes. Since the Cartwheel is a recent phenomenon, this code is especially suitable for our purposes. The results of this code are compared with those of other existing codes by Charlot (1996).

A major difference of our modeling from that of VB is in the adopted distance to the galaxy, with the value of a Hubble constant being $H_{0}=65 \mathrm{~km} \mathrm{~s}^{-1} \mathrm{Mpc}^{-1}$ instead of $100 \mathrm{~km} \mathrm{~s}^{-1} \mathrm{Mpc}^{-1}$. The need for this change comes from the realization that the results of our modeling depend on the adopted distance to the galaxy. For example, choice of $H_{0}=50 \mathrm{~km} \mathrm{~s}^{-1} \mathrm{Mpc}^{-1}$ instead of $H_{0}=100 \mathrm{~km} \mathrm{~s}^{-1} \mathrm{Mpc}^{-1}$ would double the linear diameter of the Cartwheel's disk. The time it takes for the density wave of constant propagation velocity to cross the Cartwheel's disk would grow accordingly, which will inevitably influence the colors of densitywave-born stellar populations in each radial annulus of the Cartwheel's disk. There are distinct signs of convergence between different estimates of a Hubble constant to the value of $H_{0}=65 \pm 10 \mathrm{~km} \mathrm{~s}^{-1} \mathrm{Mpc}^{-1}$ (Binney \& Merrifield 1998). Therefore, in this work we adopt $H_{0}=65 \mathrm{~km} \mathrm{~s}^{-1} \mathrm{Mpc}^{-1}$, which assumes $140 \mathrm{Mpc}$ for the distance to the Cartwheel galaxy.

We consider several propagation velocities of the outer ring $\left(v_{\text {ring }}\right)$ starting from $25 \mathrm{~km} \mathrm{~s}^{-1}$ and ending with $120 \mathrm{~km} \mathrm{~s}^{-1}$. We focus on three values, namely $25 \mathrm{~km} \mathrm{~s}^{-1}, 55 \mathrm{~km} \mathrm{~s}^{-1}$, and $90 \mathrm{~km} \mathrm{~s}^{-1}$ as implied by the measurements of gas expansion velocity by Amram et al. (1998), Higdon (1996), and Fosbury $\&$ Hawarden (1977). For each value of propagation velocity we explore a wide range of metallicities from $z=z_{\odot} / 20$ to $z=2 z_{\odot}$ in order to achieve a better agreement between the model and observed photometry. The properties of the pre-collision stellar disk are chosen typical for the late-type Freeman disks, with the $V$-band central surface brightness $\mu_{V}^{0}=21.0 \mathrm{mag} \mathrm{arcsec}^{-2}$ and the scale length $R_{0}=3 \mathrm{kpc}$ respectively. In this paper we do not model the colors of the Cartwheel's inner ring and the nucleus, as it requires additional assumptions on the star formation history in the Cartwheel (see VB for details).

The Cartwheel's and the model colors/indices obtained for the outer ring propagation velocity of $25 \mathrm{~km} \mathrm{~s}^{-1}$ and $90 \mathrm{~km} \mathrm{~s}^{-1}$ are shown in Fig. 1. Open triangles in Figs. 1a and 1b represent the model radial $B-V / V-K$ color gradients and $Q_{B V K}$ combined color indices obtained for $v_{\text {ring }}=25 \mathrm{~km} \mathrm{~s}^{-1}$, while the open squares indicate the corresponding values obtained for $v_{\text {ring }}=90 \mathrm{~km} \mathrm{~s}^{-1}$. For the latter velocity, the model colors of three outermost annuli are indicated in Fig. 1a by Arabic numbers. The filled triangles with error bars represent the radial $B-V / V-K$ color gradients and $Q_{B V K}$ combined color indices of the Cartwheel ring galaxy. The Cartwheel's colors were obtained by Marcum et al. (1992) for nine annuli and the nucleus (see their Plate I) and numbered in Fig. 1a according to radius, beginning with the inner ring (annulus I) and ending with the outer ring (annuli VIII-IX).

The fast outer ring can nicely reproduce the Cartwheel's $Q_{B V K}$ indices, if metallicity gradient of star-forming gas ranges from $z=z_{\odot}$ in the inner parts (annuli II-IV) to $z=z_{\odot} / 3.7$ in the outer parts (annuli V-IX). The $Q_{B V K}$ indices minimize the uncertainties in the observed $B-V / V-K$ colors introduced by dust 
extinction. Hence, a good correspondence between the model and the Cartwheel's $Q_{B V K}$ indices indicates that the difference in each annulus between the model $B-V / V-K$ colors shown in Fig. 1a by the open squares and the Cartwheel's $B-V / V-K$ colors might be due to dust extinction in the Cartwheel's disk. The value of color excess $E(B-V)$ implied by this difference can be used to estimate the surface densities of gas in each annulus using standard gas-to-dust ratio (Bohlin et al. 1978). The estimated surface densities of gas are below the detection upper limits for each annulus, which indicates that internal extinction is indeed responsible for the difference between the Cartwheel's $B-V / V-K$ colors and the model colors shown in Fig. 1a by the open squares.

On the other hand, the slow outer ring cannot reproduce the Cartwheel's $Q_{B V K}$ indices. Disagreement between the model and Cartwheel's indices is less severe, if metallicity gradient of star-forming gas ranges from $z=z_{\odot}$ in the inner parts (annuli II-IV) to $z=z_{\odot} / 3.7$ in the outer parts (annuli V-IX). Nevertheless, the model $Q_{B V K}$ indices shown by the open triangles in Fig. 1b lie beyond the observed limits, which indicates that the difference in each annulus between the model $B-V / V-K$ colors shown in Fig. 1a by the open triangles and the Cartwheel's colors cannot be attributed to dust extinction in the Cartwheel's disk.

We considered other propagation velocities of the outer ring and found that the Cartwheel's $B-V / V-K$ colors and $Q_{B V K}$ color indices could be acceptably reproduced for $55 \mathrm{~km} \mathrm{~s}^{-1} \leq v_{\text {ring }} \leq 120 \mathrm{~km} \mathrm{~s}^{-1}$. The best-fit value is $v_{\text {ring }}=90 \mathrm{~km} \mathrm{~s}^{-1}$. Stellar populations born in each annulus by a slower wave are older than those born by a faster wave. Strong dependence of $B-V / V-K$ colors on the age of young stellar populations allows us to constrain the propagation velocity of the Cartwheel's outer ring.

Three assumptions can possibly influence our color modeling results: the assumed properties of the pre-collision stellar disk, the adopted value of a Hubble constant, and the population synthesis model used in color gradient modeling.

a) The pre-collision stellar populations. To understand how the model $B-V / V-K$ colors depend on the properties of the pre-collision disk, we gradually reduced $\mu_{V}^{0}$ down to $23.5 \mathrm{mag}$ and increased $R_{0}$ up to $5 \mathrm{kpc}$ imitating transition from the latetype galactic disk to the low surface brightness (LSB) disk. Again, for $v_{\text {ring }}<50 \mathrm{~km} \mathrm{~s}^{-1}$ the model colors and indices totally disagree with those of the Cartwheel galaxy. The acceptable agreement between the model and the Cartwheel's radial color gradients and color indices is found for higher propagation velocities of $90 \mathrm{~km} \mathrm{~s}^{-1}$, though the model $Q_{B V K}$ indices tend to lie systematically above the observed values. This again favors to the pre-collision Cartwheel being a normal late-type galaxy rather than a LSB galaxy (VB).

b) Distance to the Cartwheel. We recomputed the model colors for a Hubble constant of $H_{0}=100 \mathrm{~km} \mathrm{~s}^{-1} \mathrm{Mpc}^{-1}$, which assumed $90 \mathrm{Mpc}$ for the distance to the Cartwheel galaxy. The adopted distance to the Cartwheel affects the model radial color gradients. A general tendency is that the acceptable agreement between the model and the Cartwheel's $B-V / V-K$ colors and $Q_{B V K}$ indices can now be achieved for lower values of the ring propagation velocity, more specifically, for $v_{\text {ring }} \geq 40 \mathrm{~km} \mathrm{~s}^{-1}$.
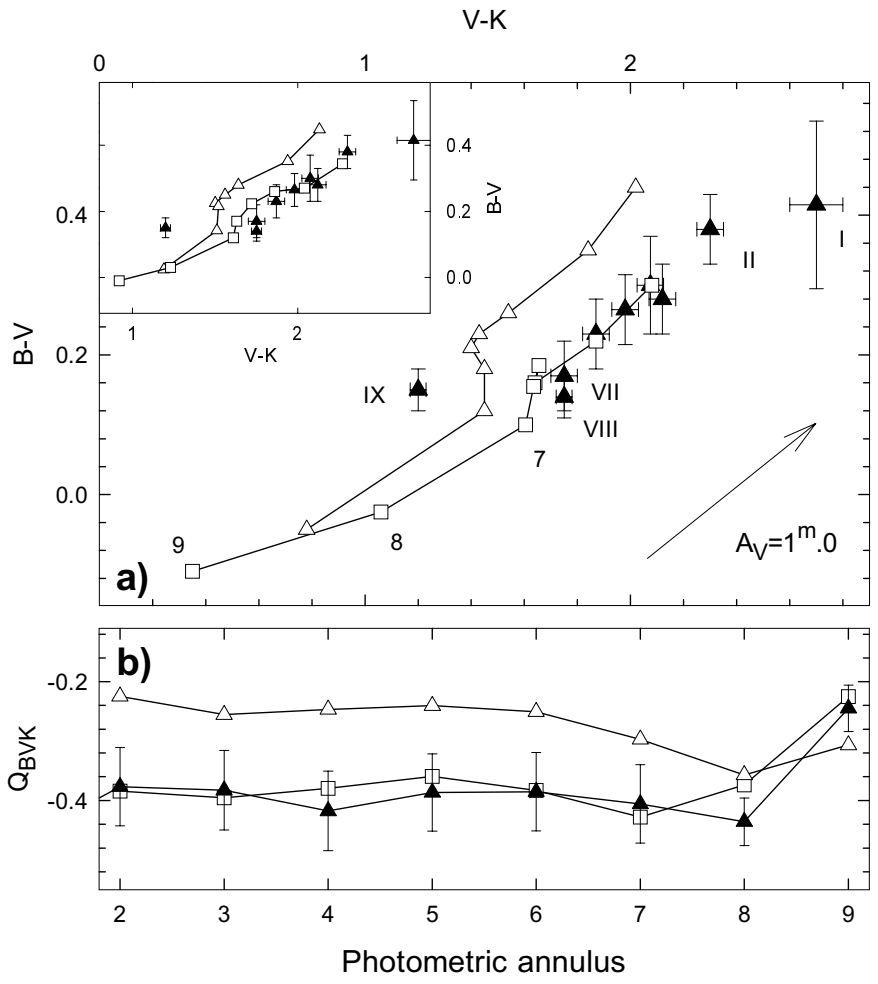

Fig. 1. The radial profiles of a) $B-V / V-K$ color gradients and b) the $Q_{B V K}$ combined color indices. The open squares and the open triangles show the model profiles obtained for the outer ring propagation velocity of $90 \mathrm{~km} \mathrm{~s}^{-1}$ and $25 \mathrm{~km} \mathrm{~s}^{-1}$ respectively. The filled triangles with error bars indicate a) the measured $B-V / V-K$ radial color gradients in the Cartwheel's disk and b) the $Q_{B V K}$ color indices calculated from the measured $B-V / V-K$ colors of the Cartwheel's disk. The insert shows the results of color modeling with Starburst99 (Leitherer et al. 1999).

The best-fit value is now $v_{\text {ring }}=60 \mathrm{~km} \mathrm{~s}^{-1}$. For the ring propagation velocities higher than $90 \mathrm{~km} \mathrm{~s}^{-1}$, the correspondence between the model and the Cartwheel's colors/indices of the four outermost annuli worsens.

c) Population synthesis model. We tested our results of color gradient modeling with another population synthesis code, namely Starburst99 (Leitherer et al. 1999). This code was developed for synthesizing observable quantities in starburst galaxies. Since the Cartwheel harbors a moderate burst of star formation $\left(S F R \approx 67 M_{\odot} \mathrm{yr}^{-1}\right)$, Starburst99 is best suited for comparison with the code assumed in this work (Mayya 1995). The results of color modeling with Starburst 99 are plotted in the insert of Fig. 1a for both the fast outer ring $\left(v_{\text {ring }}=90 \mathrm{~km} \mathrm{~s}^{-1}\right.$, the open squares $)$ and the slow outer ring $\left(v_{\text {ring }}=25 \mathrm{~km} \mathrm{~s}^{-1}\right.$, the open triangles). It is clearly evident that the fast outer ring can well reproduce the Cartwheel's colors. On the contrary, the slow outer ring cannot reproduce the Cartwheel's optical and near-infrared colors. Starburst99 uses a new atmospheric model of Lejeune et al. (1997) and the latest Geneva evolutionary models, which are different from those used by Mayya in his population synthesis code (Mayya 1995). Nevertheless, the results of color modeling with both codes are very similar. Hence, our main conclusions are not affected by the population synthesis model assumed in this work. 
Direct estimate of the Cartwheel's outer ring propagation velocity from the color gradient modeling performed in this section is problematic since our results are sensitive to the assumed value of a Hubble constant. Nevertheless, our modeling indicates that the propagation velocity of the Cartwheel's outer ring cannot be lower than $40 \mathrm{~km} \mathrm{~s}^{-1}$ for any reasonable value of a Hubble constant. Determining the ring propagation velocity may be in fact even more difficult, because it may not coincide with the expansion velocity of gas in the ring. Indeed, rings are rather wave phenomena than material features (Athanassoula et al. 1997) and the difference between the ring propagation velocity and the radial expansion velocity of particles forming the ring is not totally unexpected. In the next section two dimensional numerical simulations of a Cartwheel-like ring galaxy are performed. We restrict ourselves to the planar geometry in order to find the relationship between the maximum radial expansion velocity of gas in the ring and the propagation velocity of the gas ring itself.

\section{Numerical simulations of a Cartwheel-like ring galaxy}

\subsection{Initial conditions}

An extensive study of the Cartwheel galaxy by Higdon (1995, 1996) provided important observational constrains on the parameters of our numerical model. The indicative mass of the Cartwheel, which generally represents the total mass to within a factor of about 2 , is $5.3 \times 10^{11} M_{\odot}$ for the adopted $H_{0}=$ $65 \mathrm{~km} \mathrm{~s}^{-1} \mathrm{Mpc}^{-1}$ (Higdon 1996). An estimate of the luminous stellar mass $M_{\mathrm{st}}$ in the Cartwheel is derived by multiplying its blue luminosity $L_{\mathrm{B}}=3.7 \times 10^{10} L_{\odot}$ (Higdon 1995) by a late spiral ratio $M / L_{\mathrm{B}}=3$ (Faber \& Gallagher 1979). This results in $M_{\mathrm{st}}=1.1 \times 10^{11} M_{\odot}$. The total HI mass of the Cartwheel is $2.2 \times 10^{10} M_{\odot}$ (Higdon 1996, corrected for $\left.H_{0}=65 \mathrm{~km} \mathrm{~s}^{-1} \mathrm{Mpc}^{-1}\right)$, with the total gas mass $\left(M_{\mathrm{HI}}+M_{\mathrm{He}}\right)$ amounting to $3 \times 10^{10} M_{\odot}$. The Cartwheel galaxy thus appears to be halo-dominated. This assumption was also used by Appleton \& Higdon (1993) in their hydrodynamic models of the Cartwheel galaxy.

We consider a Cartwheel-like galaxy consisting of a selfgravitating thin gaseous disk of $M_{\mathrm{d}}=3 \times 10^{10} M_{\odot}$ and a rigid halo of $M_{\mathrm{h}}=5 \times 10^{11} M_{\odot}$. Marcum et al. (1992) found no evidence for old pre-collision stellar populations in the outer parts of the Cartwheel. Numerical modeling of the radial $B-V / V-K$ color gradients confirmed that the pre-collision stellar populations dominate only in the inner parts of the Cartwheel (VB). Moreover, our numerical simulations indicate that stellar density waves propagate only 2-3 disk scale lengths and become significantly less defined as compared to gas density waves, the result also found in numerical simulations by Hernquist \& Weil (1993). Hence, we expect that the stellar density wave would have little effect on the dynamics of gas at the radii of the Cartwheel's outer ring.

The radial distribution of gas in the pre-collision Cartwheel is unknown. Our modeling of radial color gradients measured in the Cartwheel's disk (VB) suggested that this distribution most probably showed a mild exponential decline with a surface density contrast between the nucleus and the position of the outer ring being a factor of 3.5. More importantly, the lack of old stellar component at the outer parts of the Cartwheel implies that the surface density of gas in the pre-collision Cartwheel was below the critical surface density for gravitational instability everywhere except probably the innermost radii. The critical surface density is defined as

$\Sigma_{\text {crit }}=0.7 \frac{v_{\mathrm{s}} k}{\pi G}$

where $v_{\mathrm{s}}$ is the speed of sound and $k$ is the epicyclic frequency (Martin \& Kennicutt 2001).

Initially, the rotating gaseous disk is balanced by selfgravity of the disk, the radial pressure gradient, and gravity of the spherically symmetric rigid halo

$\frac{v_{\text {cir }}^{2}}{r}=\frac{1}{\Sigma_{\mathrm{g}}} \frac{\mathrm{d} P}{\mathrm{~d} r}+\frac{\mathrm{d}}{\mathrm{d} r}\left(\Phi_{\mathrm{d}}+\Phi_{\mathrm{h}}\right)$,

where $v_{\text {cir }}$ is the rotational velocity, $P$ is the gas pressure, $\Phi_{\mathrm{h}}$ is the halo potential, and $\Phi_{d}$ is the potential of gaseous disk defined in the polar coordinates as

$$
\begin{aligned}
\Phi_{\mathrm{d}}(r, \phi)= & -G \int_{R_{\text {in }}}^{R_{\text {out }}} r^{\prime} \mathrm{d} r^{\prime} \\
& \times \int_{0}^{2 \pi} \frac{\Sigma_{\mathrm{g}}\left(r^{\prime}, \phi^{\prime}\right) \mathrm{d} \phi^{\prime}}{\sqrt{r^{2}+r^{\prime 2}-2 r r^{\prime} \cos \left(\phi-\phi^{\prime}\right)}}
\end{aligned}
$$

We adopt an isothermal equation of state $\left(T=10^{4} \mathrm{~K}\right)$, which is an usual approximation used for modeling the interstellar medium in disk galaxies (Mihos \& Hernquist 1994). Struck (1997) has demonstrated that the ring structure and dynamics in the isothermal and non-isothermal simulations are similar. We also tried an adiabatic equation of state and found little difference in the first ring structure and dynamics, though the second ring structure and dynamics showed substantial differences with respect to the isothermal case.

A slightly modified form of rotation curve in a softening point-mass potential is adopted

$v_{\text {cir }}(r)=\frac{v_{0} r^{0.9}}{\left(r^{2}+R_{0}^{2}\right)^{0.5}}$

where $R_{0}$ is the effective halo radius. The power index 0.9 allows us to model a Keplerian decline in rotation curves at larger radii. Parameters of the rotation curve (Eq. (4)) are chosen so that the halo mass

$M_{\mathrm{h}}=\left.\frac{R_{\mathrm{out}}^{2}}{G} \frac{\mathrm{d} \Phi_{\mathrm{h}}}{\mathrm{d} r}\right|_{r=R_{\mathrm{out}}}$

is equal to $5 \times 10^{11} M_{\odot}$. As the rotational curve is fixed by Eq. (4) and the disk potential $\Phi_{d}$ is determined by numerically integrating Eq. (3), the gradient of halo potential $\Phi_{\mathrm{h}}$ can be calculated using Eq. (2). Parameters used in numerical simulations are listed in Table 1. 
Table 1. Model parameters of a Cartwheel-like galaxy.

\begin{tabular}{ll}
\hline \hline Parameter & Value \\
\hline Inner disk radius $\left(R_{\text {in }}\right)$ & $0.2 \mathrm{kpc}$ \\
Outer disk radius $\left(R_{\text {out }}\right)$ & $34 \mathrm{kpc}$ \\
Disk scale length $\left(r_{0}\right)$ & $20 \mathrm{kpc}$ \\
Central surface density of gas $\left(\Sigma_{\mathrm{g}}(0)\right)$ & $23.5 M_{\odot} \mathrm{pc}^{-2}$ \\
Gas temperature $(T)$ & $10^{4} \mathrm{~K}$ \\
Disk mass $\left(M_{\mathrm{d}}\right)$ & $3 \times 10^{10} M_{\odot}$ \\
Halo mass $\left(M_{\mathrm{h}}\right)$ & $5 \times 10^{11} M_{\odot}$ \\
Mass of a companion $\left(M_{\mathrm{c}}\right)$ & $1.5 \times 10^{11} M_{\odot}$ \\
Companion's softening parameter $(\epsilon)$ & $5 \mathrm{kpc}$ \\
\hline
\end{tabular}

\subsection{Computational techniques}

We use the ZEUS-2D numerical hydrodynamics code incorporating a self-gravitating thin gaseous disk and a fixed spherically symmetric halo potential. A usual set of isothermal hydrodynamics equations in the polar coordinates $(r, \phi)$ is solved using the method of finite-differences with a time-explicit, operator split solution procedure explained in detail in Stone \& Norman (1992). The interaction is treated by computing the gravitational force exerted on the disk by the softened pointmass potential of a companion galaxy. Equations of motion of the companion in the combined gravitational potential of the halo and the gaseous disk are numerically solved using the Runge-Kutta scheme. The gravitational potential of the thin disk (Eq. (3)) is evaluated numerically using the fast Fourier transform (Binney \& Tremaine 1987). The code was verified against a standard set of test problems described in Stone \& Norman (1992).

\subsection{Results of modeling}

We attempt to find the relationship between the propagation velocity of ring gas density wave and the maximum radial expansion velocity of gas in the wave generated by a near central passage of a companion galaxy through the disk of a Cartwheel-like galaxy. We have chosen the model parameters typical for the Cartwheel galaxy in order to compare the results of numerical simulations with those of Sect. 2. By varying the initial rotation curve, the mass of a companion, and the impact point we try to reproduce the morphology, kinematics, and radial distribution of gas in the Cartwheel's disk. Below we briefly describe the parameter space investigated in our simulations.

Initial rotation curve. We consider three initial rotation curves shown in Fig. 2 and find that the Cartwheel's HI morphology and kinematics are best reproduced for A 2 curve. A1 curve produce too small a spacing between the inner and outer rings as compared to that of the Cartwheel, while A3 curve does not develop the inner ring at all.

Mass of a companion. We consider a wide range of companion galaxy masses from $4 \times 10^{10} M_{\odot}$ to $3 \times 10^{11} M_{\odot}$, which is roughly $7.5 \%-55 \%$ of the Cartwheel's total mass. The indicative mass of a companion G3, most massive in the Cartwheel's group (Higdon 1996), is $M_{\mathrm{G} 3}=4 \times 10^{10} M_{\odot}$. This mass is not enough to reproduce the radial surface density profiles of $\mathrm{HI}$ in the Cartwheel. Such a low-mass companion gives a weak first response, with less than a $20 \%$ enhancement in gas densities over the unperturbed values. At the time when the first ring reaches $24 \mathrm{kpc}$ radius (the current location of the Cartwheel's outer ring), the actual mass of gas locked in the ring is only $25 \%$ of the total gas mass, while in the Cartwheel the outer ring contains about $85 \%$ of total HI (Higdon 1996). The low mass companion does not reproduce the observed spacing between the inner and the outer Cartwheel's rings, with the radius of the model second ring being almost twice bigger than that of the Cartwheel's inner ring.

We find that companions with masses no less than $25 \%$ of the Cartwheel's total mass can acceptably reproduce the HI distribution in the Cartwheel's disk. In our simulations the companion's mass is $1.5 \times 10^{11} M_{\odot}$, which is roughly $30 \%$ of the total mass of the Cartwheel. A $30 \%$ companion generates stronger response, with more than a $100 \%$ enhancement in gas surface densities over the unperturbed values. There are no companions to the Cartwheel large enough to fit the companion's mass we determine. The companion's optical and HI line widths give mass estimates that are only $\sim 6 \%$ of the Cartwheel's (Davies \& Morton 1982; Higdon 1996). However, determining accurate galaxy masses is difficult enough in strongly perturbed galaxies like the Cartwheel's companions. Passing through the Cartwheel's disk causes the companion to lose mass due to tidal and ram pressure stripping. Indeed, Higdon (1996) reported HI plumes stretching from the Cartwheel toward G3 companion. Ring galaxy simulations by Tsuchiya et al. (1998) found that massive companions $(25 \%$ of ring galaxy's mass) can loose around 35\% of their halos, or nearly a third of their total mass, due to the interaction. The gas disks in massive companions were also greatly truncated ( $R_{\text {gas }}<50 \%$ of original radius). If much of the companion's mass resided in a halo, one would in fact significantly underestimate its total mass. The apparent low masses of the companion galaxies are a problem, but it is likely to be a consequence of the ring galaxy formation process. More work is clearly needed in estimating masses of ring galaxy companions to see if this is a general result.

Impact points, impact angles, and relative velocities. In our simulations the companion placed initially at a distance of $35 \mathrm{kpc}$ above the Cartwheel's plane, which was roughly $150 \%$ of the Cartwheel's radius, was given an initial relative velocity of $80 \mathrm{~km} \mathrm{~s}^{-1}$ toward the Cartwheel. Varying the initial distances from the axis of the Cartwheel, we obtained a wide range of impact points starting from a nearly bull's-eye collision $\left(R_{\text {impact }}=0.1 \mathrm{kpc}\right)$ and ending with an essentially offcenter collision $\left(R_{\text {impact }}=10 \mathrm{kpc}\right)$. The former produces symmetric rings with negligible azimuthal variations in gas surface densities around the ring, while the latter results in crescent or spiral-like density waves. Neither of these ultimate cases resembles the actual HI distribution in the Cartwheel's outer ring. We find that the Cartwheel's HI kinematics and morphology are best reproduced for a slightly off-center $R_{\text {impact }}=$ $2.5 \mathrm{kpc}$ collision. The relative velocity of the companion at the time of impact is $480 \mathrm{~km} \mathrm{~s}^{-1}$. We also considered other distances above the Cartwheel's plane and other relative velocities as the initial parameters of the companion. For the initial 


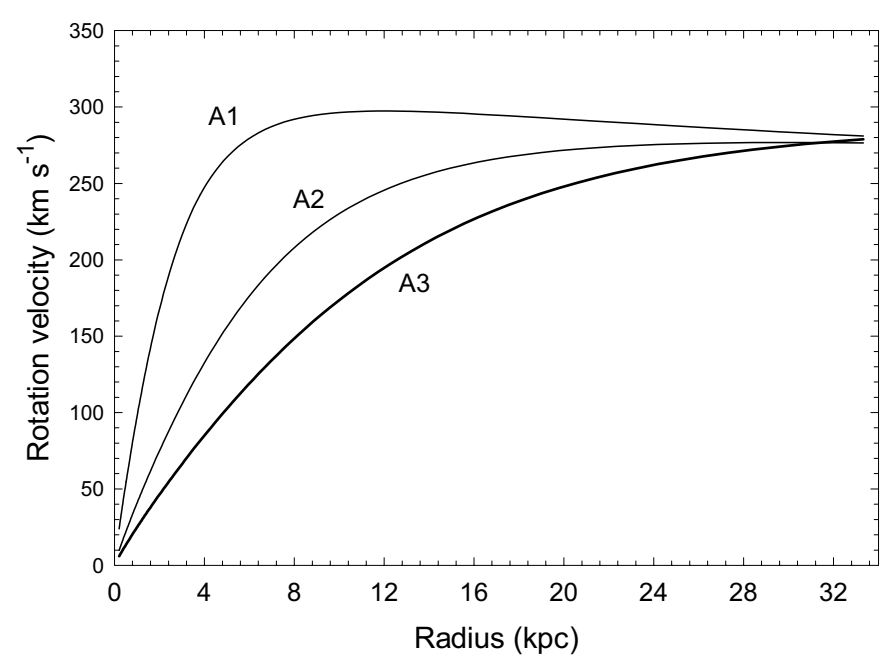

Fig. 2. Rotation curves of pre-collision gaseous disk generated using Eq. (4). Parameters determining rotation curves are: $v_{0}=375$ and $R_{0}=4$ (marked "A1"), $v_{0}=382$ and $R_{0}=10$ (marked "A2"), $v_{0}=420$ and $R_{0}=18$ (marked "A3"). The Cartwheel's outer ring is at $24 \mathrm{kpc}$ $\left(H_{0}=65 \mathrm{~km} \mathrm{~s}^{-1} \mathrm{Mpc}^{-1}\right)$.

distance of $70 \mathrm{kpc}$ and initial relative velocity of $80 \mathrm{~km} \mathrm{~s}^{-1}$ toward the Cartwheel, the relative velocity at the time of impact was $v_{\text {relative }}$ (impact) $=540 \mathrm{~km} \mathrm{~s}^{-1}$. For the initial distance of $35 \mathrm{kpc}$ and initial relative velocity of $380 \mathrm{~km} \mathrm{~s}^{-1}$ to-

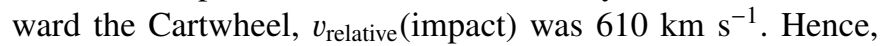
$v_{\text {relative }}(\mathrm{impact})$ is largely determined by the parameters of the Cartwheel. Variations of $15 \%$ in the relative velocity affect insignificantly the gas dynamics after the collision. Angles of impact from the axis of the Cartwheel are $5-15^{\circ}$ in all considered cases. We have not made special efforts to model highly inclined collisions.

Figure 3 shows the model velocity field of gas superimposed on the contour plot of gas surface density $\Sigma_{\mathrm{g}}$ obtained for A2 curve and model parameters listed in Table 1. The contrast between $\Sigma_{\mathrm{g}}$ in the outer ring and the inter-ring region is in agreement with observations. $\Sigma_{\mathrm{g}}$ between the rings in Fig. 3 never exceed $7 M_{\odot} \mathrm{pc}^{-2}$ and falls below $5 M_{\odot} \mathrm{pc}^{-2}$ behind the densest regions in the outer ring. Maximum and average $\Sigma_{\mathrm{g}}$ around the outer ring are 18.5 and $15 M_{\odot} \mathrm{pc}^{-2}$, however the observed high density condensations up to $60 M_{\odot} \mathrm{pc}^{-2}$ are not found in simulations. $\Sigma_{\mathrm{g}}$ is below $7 M_{\odot} \mathrm{pc}^{-2}$ beyond the outer ring. High density clumps are found in the inner ring and central regions, with $\Sigma_{\mathrm{g}} \approx 60-100 M_{\odot} \mathrm{pc}^{-2}$ and even higher. Development of such high density clumps is interesting, since high densities and low temperatures are thought to favor $\mathrm{HI} \rightarrow \mathrm{H}_{2}$ transitions in giant gas clouds. Indeed, Horrellou et al. (1998) detected a substantial amount of molecular hydrogen in the Cartwheel's inner part. For the solar metallicity implied by color gradient modeling in Sect. $2, \mathrm{H}_{2}$ mass within the inner ring amounts to $2.1 \times 10^{9} M_{\odot}$ according to Horellou et al. (1998). In our simulations, the inner ring contains $\leq 10 \%$ of the gas mass or $\leq 3 \times 10^{9} M_{\odot}$, which is roughly consistent with the detected $\mathrm{H}_{2}$ mass. In Sect. 3.1 we neglected a possible contribution of molecular hydrogen to the gas mass of the Cartwheel as it was only a small fraction to the total mass of

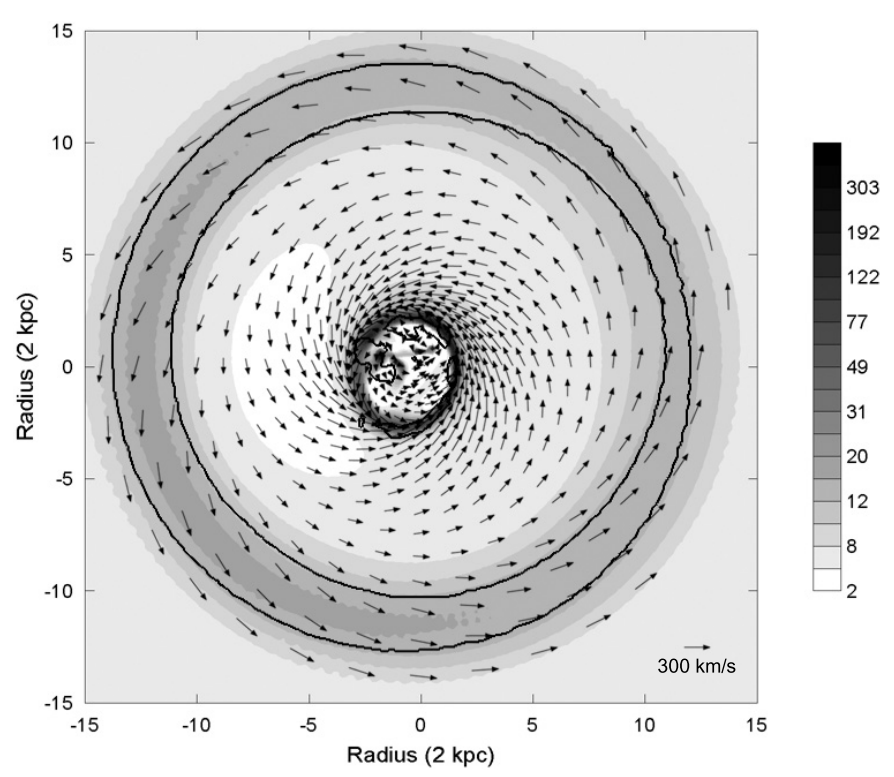

Fig. 3. The model velocity field of gas superimposed on the contour plot of gas surface density $\Sigma_{\mathrm{g}}$ obtained for A2 curve and model parameters listed in Table 1. The solid line outlines the regions where $\Sigma_{\mathrm{g}}$ exceeds the critical surface density for gravitational instability defined by Eq. (1). The scale bar is in $M_{\odot} \mathrm{pc}^{-2}$.

the Cartwheel galaxy $(\leq 1 \%)$ and would not affect noticeably the companion's dynamics.

While the inner ring in Fig. 3 has on average higher surface densities of gas than the outer ring, it contains less than $10 \%$ of the total disk mass. On the contrary, the outer ring in Fig. 3 comprises more than $50 \%$ of the total disk mass. No obvious spokes were obtained for either of three initial rotation curves. Indeed, HI observations of Higdon (1996) have shown that the Cartwheel's spokes are HI poor. The solid line in Fig. 3 outlines the regions where $\Sigma_{\mathrm{g}}$ exceeds the critical surface density for gravitational instability defined by Eq. (1). The regions where star formation is supposed to occur correlate with the regions of maximum $\Sigma_{\mathrm{g}}$ in the outer ring. This is not the case for the Cartwheel, where a pronounced anticorrelation between $\Sigma_{\mathrm{HI}}$ and $\mathrm{H} \alpha$ surface brightness was found (Higdon 1996). Among other reasons such as a blow-out in giant supershells formed by multiple supernova explosions, this might be explained as the consumption of the gas supply by the starburst. Inclusion of star formation processes in the code is required to check this hypothesis.

The velocity field in Fig. 3 shows all basic features of HI kinematics found in the Cartwheel: (1) a general increase in expansion velocity of gas for $R>16 \mathrm{kpc}$, (2) inflow of gas towards the inner ring for $R<16 \mathrm{kpc}$. The flow of gas inside the inner ring is complicated. However, a general expansion of gas is clearly seen in this innermost region. We determine the relationship between the propagation velocity of the outer ring $v_{\text {ring }}$ (i.e. the propagation velocity of gas density wave) and maximum radial expansion velocity of gas $v_{\text {gas }}$ in the outer ring at different ring expansion phases. The regions of maximum density enhancements in the outer ring are used to trace the outer ring position during the runs. We start from very early times when the outer ring was only $\approx 6 \mathrm{kpc}$ in radius and stop at the 


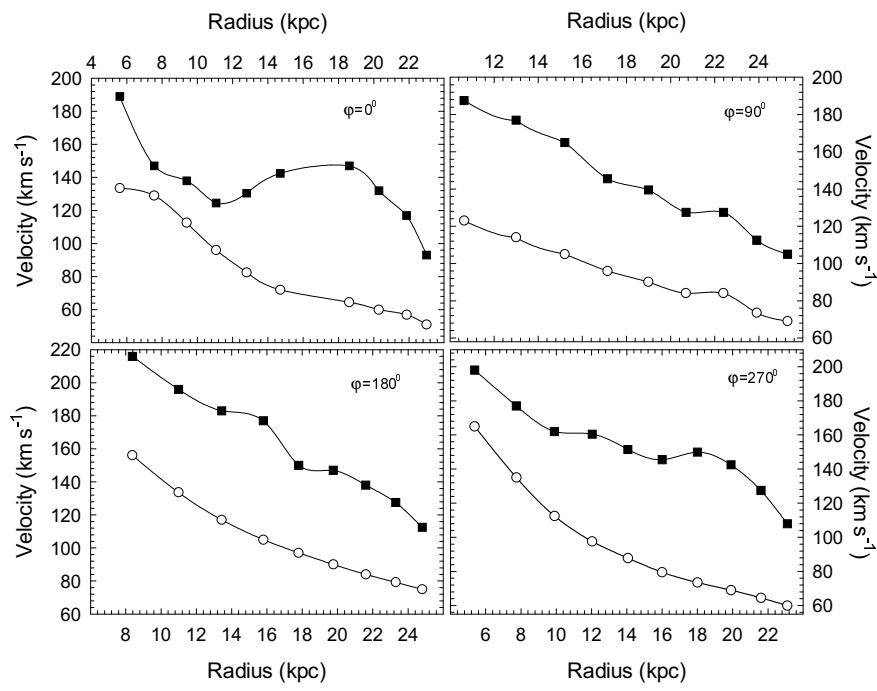

Fig. 4. The propagation velocities of the outer ring (the filled squares) and the maximum expansion velocities of gas in the ring (the open circles) traced along four different polar angles as indicated in each frame. The propagation velocities of the outer ring are systematically higher than the maximum expansion velocities of gas in the ring.

outer ring current position of $\approx 24 \mathrm{kpc}$. Due to obvious asymmetric structure of $\Sigma_{\mathrm{g}}$ in Fig. 3, these velocities were traced along four different polar angles of $0^{\circ}, 90^{\circ}, 180^{\circ}$, and $270^{\circ}$. The results are shown in Fig. 4. The propagation velocities of the outer ring (the filled squares) are systematically higher than the maximum radial expansion velocities of gas in the ring (the open circles). Both velocities generally decline as the outer ring propagates outwards. At the time when the outer ring reaches its current location at the radius of $24 \mathrm{kpc}, v_{\text {ring }}$ and $v_{\text {gas }}$ slow down on average to $102 \mathrm{~km} \mathrm{~s}^{-1}$ and $65 \mathrm{~km} \mathrm{~s}^{-1}$ respectively. However, sinusoidal azimuthal variations in the values of both velocities are evident in Fig. 4. The value of $v_{\text {ring }}=102 \mathrm{~km} \mathrm{~s}^{-1}$ is consistent with the estimates of Sect. 2, where best agreement between the the model and observed radial $B-V / V-K$ color gradients was found for $v_{\text {ring }} \geq 90 \mathrm{~km} \mathrm{~s}^{-1}$. The value of $v_{\text {gas }}=65 \mathrm{~km} \mathrm{~s}^{-1}$ is close to the upper estimate of Higdon (1996) based on HI kinematics in the Cartwheel's outer ring. The value of $v_{\text {gas }}=13-30 \pm 10 \mathrm{~km} \mathrm{~s}^{-1}$ obtained by Amram et al. (1998) using $\mathrm{H} \alpha$ kinematics of ionized gas stands apart. It is known that dynamics of different interstellar phases may considerably differ in disk galaxies. Inclusion of multiphase gas dynamics and star formation in the code is needed to determine the dynamical properties of ionized gas in ring galaxies.

We performed a series of computations with different values of $M_{\mathrm{d}}, M_{\mathrm{h}}, M_{\mathrm{c}}, r_{0}$, and different initial rotation curves. In Figs. 5a-c we plot the maximum expansion velocities of gas in the ring at the radii of 12 (the upper frame), 18 (the intermediate frame), and $22 \mathrm{kpc}$ (the lower frame) as a function of the azimuthally averaged propagation velocities of the first ring at the same radii. Parameters of each run labeled by Arabic numbers in Fig. 5a are listed in Table 2. Impact points and impact angles vary slightly in the range $1.5-3 \mathrm{kpc}$ and $5-15^{\circ}$ respectively. We find that the inequality $v_{\text {ring }}>v_{\text {gas }}$ holds in all cases, though the resulted morphology and kinematics of gas may hardly resemble those of the Cartwheel. Both velocities are generally higher at the smaller radii and they decline at the larger radii. Higher mass companions produce a stronger response with higher ring propagation velocities and maximum radial expansion velocities of gas in the ring, thus leaving the relation between them unchanged. It appears that the $v_{\text {ring }}>v_{\text {gas }}$ inequality holds for the first gas rings triggered by the passage of a companion through the disk of a halo-dominated target galaxy. The leastsquares fit to a linear function $v_{\text {gas }}=k v_{\text {ring }}$ shown in each panel of Fig. 5 by a dash-dotted line gives $k=0.67$ for the rings of $12 \mathrm{kpc}, k=0.55$ for the rings of $18 \mathrm{kpc}$, and $k=0.51$ for the rings of $24 \mathrm{kpc}$. A linear function $v_{\text {gas }}=v_{\text {ring }}$ is plotted for comparison in each panel of Fig. 5 by a dashed line. We also considered gas rings in ring galaxies with comparable disk and halo masses, for which we obtained an average value of $k=0.8$ for the rings of 12,18 , and $24 \mathrm{kpc}$. In higher order resonant rings (i.e. the second rings) the flow is more complicated and $v_{\text {ring }}>v_{\text {gas }}$ inequality is no more valid. The $v_{\text {ring }}>v_{\text {gas }}$ inequality contradicts the predictions of a simple kinematical model of ring galaxy formation, which argues that a determination of the expansion velocity of an individual star or HII region in the first ring gives a good indication of the ring propagation velocity (Toomre 1978).

Higdon (1996) reported the narrow HI line widths and the absence of strong shear in the Cartwheel's outer ring. He concluded that the outer ring appeared to initiate star formation activity primarily through concentration of the interstellar medium in the ring rather than through high-speed disruptive cloud collisions $\left(v_{\text {relative }} \geq 100 \mathrm{~km} \mathrm{~s}^{-1}\right.$ ) as advocated by Olson \& Kwan (1990) for starburst activity in interacting/merging galaxies. Apparent lack of molecular hydrogen in the outer ring (Horellou 1995) argues against compact dense clouds. Hence, we have chosen a smooth initial gas distribution for the precollision Cartwheel rather than a clumpy medium. In the wider context of a disparity in ring propagation velocity versus gas expansion velocity in ring galaxies, the $v_{\text {ring }}>v_{\text {gas }}$ inequality has to be proved for a clumpy gas distribution. In this connection, sticky particle codes (Combes \& Gerin 1985) could be very useful. We note here that N-body simulations of stellar rings in ring galaxies by Athanassoula et al. (1997) have also shown that the expansion velocity of the first stellar ring is usually higher than the mean velocity of stellar particles constituting the ring (their Fig. 17, the upper frame). Hence, a disparity in ring propagation velocity versus expansion velocity of gas/stars constituting the ring might be a general property of ring galaxies.

The $v_{\text {ring }}>v_{\text {gas }}$ inequality for the first gas ring in ring galaxies has an important implication. Measurements of the radial expansion velocity of gas in the Cartwheel's outer ring based on $\mathrm{HI}$ and $\mathrm{H} \alpha$ kinematics appear to underestimate the true propagation velocity of the outer ring. Moreover, the radial expansion velocity of gas itself may be underestimated. Indeed, $v_{\text {gas }}$ is derived assuming that all the gas motions are in the plane of the ring. The plane geometry neglects any non-planar component of $v_{\text {gas }}$ that may be present in warped rings. Results of numerical experiments performed by Struck (1997) indicate that the Cartwheel's disk might be slightly warped. Hence, the measurements of gas expansion velocity in the Cartwheel's outer ring may underestimate $v_{\text {gas }}$, which in turn underestimates the 


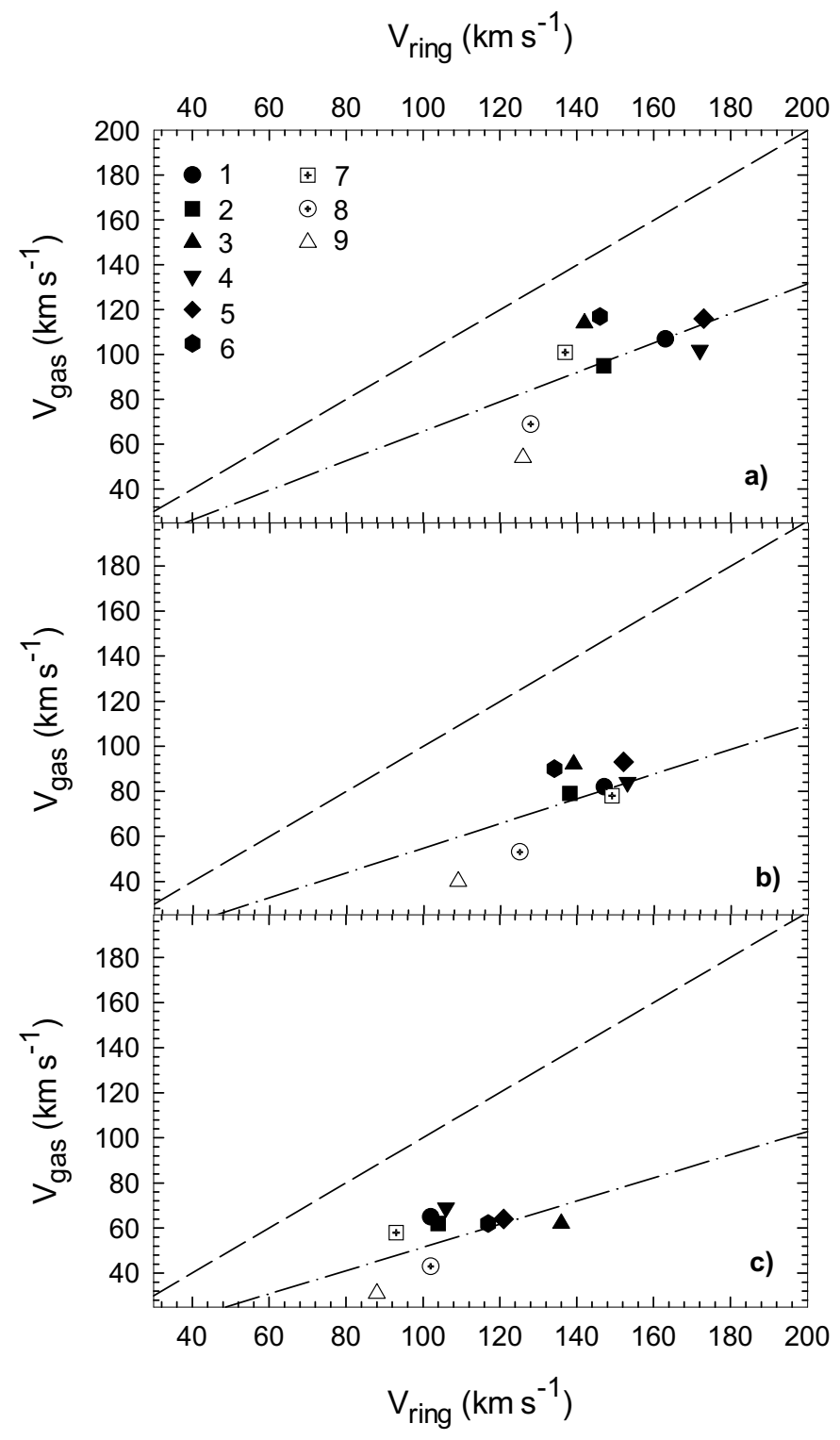

Fig. 5. The maximum expansion velocities of gas in the ring as a function of propagation velocities of the first ring at the radii of a) $12 \mathrm{kpc}$, b) $18 \mathrm{kpc}$, and c) $22 \mathrm{kpc}$. Parameters of nine runs labeled by Arabic numbers in the upper frame are listed in Table 2. The least-squares fit to a linear function $v_{\text {gas }}=k v_{\text {ring }}$ is shown in each panel by a dashdotted line. The coefficient $k$ is defined in the text. A linear function $v_{\text {gas }}=v_{\text {ring }}$ is plotted for comparison in each panel by a dashed line.

true propagation velocity of the outer ring. The same might be true for other ring galaxies. If so, ring galaxies are generally younger than they have been previously thought. If rings are younger then we can expect to see even fewer of them - they should be relatively rarer.

\section{Radial B-band, K-band, and $\mathrm{H} \alpha$ surface brightness profiles of the Cartwheel galaxy}

Marston \& Appleton (1995) found that unlike normal galaxies the azimuthally averaged radial intensity profiles in $\mathrm{H} \alpha$ and $K$-band of most ring galaxies peak at the position of the ring. Specifically, for the majority of ring galaxies the peak in the $\mathrm{H} \alpha$
Table 2. Model parameters of nine runs shown in Fig. 5.

\begin{tabular}{llllllllll}
\hline \hline $\mathrm{N}$ & $M_{\mathrm{d}}^{1}$ & $M_{\mathrm{h}}^{1}$ & $M_{\mathrm{c}}^{1}$ & $r_{0}^{2}$ & $R C^{3}$ & $v_{\text {ring }}^{4}$ & $v_{\text {gas }}^{4}$ & $v_{\text {ring }}^{5}$ & $v_{\text {gas }}^{5}$ \\
\hline 1 & 3 & 50 & 15 & 20 & $\mathrm{~A} 2$ & 163 & 107 & 102 & 65 \\
2 & 3 & 50 & 15 & 20 & $\mathrm{~A} 1$ & 147 & 95 & 104 & 62 \\
3 & 3 & 50 & 15 & 10 & $\mathrm{~A} 2$ & 142 & 114 & 136 & 62 \\
4 & 3 & 50 & 15 & 100 & $\mathrm{~A} 2$ & 172 & 102 & 105 & 68 \\
5 & 3 & 50 & 21 & 20 & $\mathrm{~A} 2$ & 173 & 116 & 121 & 64 \\
6 & 3 & 50 & 15 & 20 & $\mathrm{~A} 3$ & 146 & 117 & 117 & 62 \\
7 & 6 & 45 & 15 & 20 & $\mathrm{~A} 2$ & 137 & 101 & 93 & 58 \\
8 & 3 & 50 & 10 & 20 & $\mathrm{~A} 2$ & 128 & 69 & 102 & 43 \\
9 & 3 & 50 & 7.5 & 20 & $\mathrm{~A} 2$ & 126 & 54 & 88 & 31 \\
\hline
\end{tabular}

${ }^{1}$ In $10^{10} M_{\odot}$.

2 In kpc.

${ }^{3}$ Rotation curve as labeled in Fig. 2.

${ }^{4} v_{\text {ring }}$ and $v_{\text {gas }}$ at $12 \mathrm{kpc}$ radius.

${ }^{5} v_{\text {ring }}$ and $v_{\text {gas }}$ at $22 \mathrm{kpc}$ radius (position of the Cartwheel's outer ring).

radial profile lies at the larger radii than the peak in the $K$-band radial profile. Indeed, a standard scenario of ring galaxy formation predicts that the $K$-band and $\mathrm{H} \alpha$ peaks in radial intensity profiles of density-wave-born stellar populations are spatially separated, with the peak in $\mathrm{H} \alpha$ lying on the leading edge of the wave where most star formation takes place, and the peak in $K$-band lying progressively behind the wave as the densitywave-born stellar populations evolve (Korchagin et al. 1998).

Applying the model used to compute radial color gradients in Sect. 2, we found that the linear difference between the $K$-band and $\mathrm{H} \alpha$ peak positions depends not only on the wave velocity, but also on the metallicity of star-forming gas. Specifically, this difference is proportional to the wave velocity. For metallicities of star-forming gas below $1 / 5$ of the solar there is no peak in $K$-band at the position of the ring and the model $K$-band radial surface brightness profile shows a monotonous decline at all radii. The peak appears at higher metallicities and becomes more pronounced with increasing metallicity. In Fig. 6 we present the model azimuthally averaged radial surface brightness profiles of the Cartwheel galaxy in $K$-band (filled squares), $B$-band (open circles), and $\mathrm{H} \alpha$ (filled triangles). All model parameters of the gas density wave and pre-collision stellar populations are chosen as in Fig. 1. The model surface brightness profiles peak at/near the position of the Cartwheel's outer ring at $24 \mathrm{kpc}$. Analysis of Fig. 6 indicates that the difference between the $\mathrm{H} \alpha$ and $K$-band peak positions is expected to be around $1.1 \mathrm{kpc}\left(1.65^{\prime \prime}\right)$ for the adopted distance of $140 \mathrm{Mpc}$ and the outer ring propagation velocity of $90 \mathrm{~km} \mathrm{~s}^{-1}$. The $\mathrm{H} \alpha-B$ peak difference is $0.7 \mathrm{kpc}\left(1.05^{\prime \prime}\right)$. We note here that the underlying ring stellar density wave may influence the peak position in $K$-band. To exclude this effect, density-wave-born stellar populations must dominate the ring emission, which appears to be the case for the Cartwheel galaxy (Marcum et al. 1992, VB).

We compare our model predictions with the actual angular differences between the peaks in the azimuthally averaged $B$-band, $K s$-band and $\mathrm{H} \alpha$ radial surface brightness profiles of 


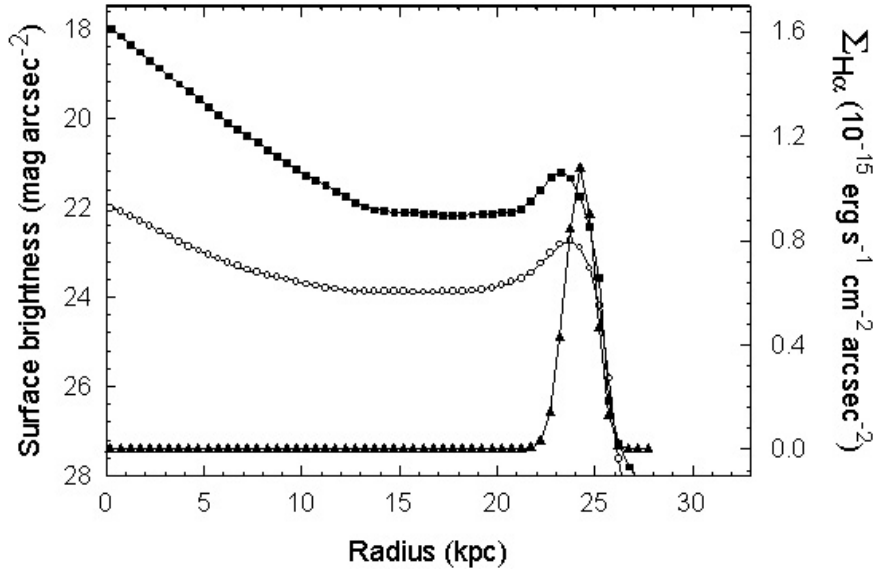

Fig. 6. The model $K$-band (filled squares), $B$-band (open circles), and $H \alpha$ (filled triangles) radial surface brightnesses of the Cartwheel ring galaxy obtained for the same model parameters as in Fig. 1. The surface brightness profiles peak at/near the position of the outer ring at $24 \mathrm{kpc}$. Difference in the peak positions at $K$-band and $\mathrm{H} \alpha$ is bigger than at $B$-band and $\mathrm{H} \alpha$.

the Cartwheel's outer ring. Images of the Cartwheel together with the calibrating frames were obtained from the public archives. The near-infrared images in $K s$-band were provided by ESO VLT archive. Images in the HST's WFPC2/F450W $(B)$ band were taken from the Hubble Space Telescope archive. Hereafter, we refer to this band as the " $B$-band". Images in $\mathrm{H} \alpha$ and "H $\alpha$-free continuum" were provided by the CFHT archive. All the data were reduced in the standard way. Since we were interested only in determining the angular difference between the peak positions in different passbands, the flux calibration was not applied. All the images were aligned using the standard MIDAS routines. The estimated accuracy of alignment is better than $0.03^{\prime \prime}$. Finally, an angular resolution of $0.76^{\prime \prime}$ was achieved for the $\mathrm{H} \alpha$ image. When the $B$ and $K s$ images are considered separately from the $\mathrm{H} \alpha$ image, a better resolution of $0.46^{\prime \prime}$ is achieved. At the adopted distance of $140 \mathrm{Mpc}$, this corresponds to a spatial resolution of $500 \mathrm{pc}$ for the $\mathrm{H} \alpha$ image and $300 \mathrm{pc}$ for the images in $K s$ - and $B$-bands.

Figure 7 shows the image of the Cartwheel galaxy in $K s$ band. The north is down and the east is to the right. As is seen in Fig. 7, the geometrical center of the outer ring does not coincide with the nucleus. To take this peculiar morphology into account, a series of elliptical annuli were constructed, with the smallest ellipse centered on the nucleus and the centers of larger ellipses slightly shifted so as the largest ellipse is centered on the geometrical center of the outer ring. These elliptical annuli were then used to determine the azimuthally averaged $K s$-band, $B$-band, and $\mathrm{H} \alpha$ radial surface brightness profiles shown in Fig. 8.

Surprisingly, we find no noticeable angular difference between the peak positions of the azimuthally averaged radial surface brightness profiles in the Cartwheel's outer ring, contrary to what is expected from our modeling (see Fig. 6). Absence of such a difference is also evident in Fig. 4 of Higdon (1995). This implies that either the outer ring velocity is too slow for this difference to be resolved or considerable inclination of the Cartwheel acts to smear out this difference. Indeed, if the Cartwheel's disk is warped (Struck 1997), then the elliptical annuli might not be a good choice for obtaining the azimuthally averaged radial profiles. Another geometry effect may also affect the azimuthally averaged profiles in considerably inclined ring galaxies. For an inclined disk of finite thickness, the lineof-sight flux comes from density-wave-born stellar populations located at different radii, and hence having different ages and different photometric properties. This line-of-sight overlapping of stellar populations of different ages may have a significant effect on the azimuthally averages surface brightness profiles, if most of the radiation flux comes from regions close to the minor axis and the line-of-sight thickness of the disk is comparable to the expected shift between the peaks in different passbands.

Rough estimates of this effect can be done by assuming that the total stars+gas surface density in the ring $\left(\Sigma_{\text {tot }}\right)$ is of order $20 M_{\odot} \mathrm{pc}^{-2}$ and using a typical value of the stellar velocity dispersion of young stellar populations $c_{z}=10 \mathrm{~km} \mathrm{~s}^{-1}$. For the case of a self-gravitating disk, the isothermal scale height of the ring $h=c^{2} / 2 \pi G \Sigma_{\text {tot }}$ is near $0.2 \mathrm{kpc}$. The vertical thickness of the outer ring $2 h$ is thus $0.4 \mathrm{kpc}$. The gravitational potential of a dark halo makes a disk thinner, but not very significantly (Bahcall 1984). At the same time, disk thickness is expected to be twice bigger for interacting galaxies (Reshetnikov $\&$ Combes 1997). Results of numerical experiments also indicate that the Cartwheel's outer ring might be thick and slightly warped (Struck 1997).

Our model predicts that the linear difference between the $\mathrm{H} \alpha$ and $K$-band peaks is of order of a kiloparsec and this value is thus comparable to the line-of-sight thickness of the Cartwheel outer ring for the adopted inclination of $41^{\circ}$ (Higdon 1996). Hence, we expect that both the line-of-sight overlapping and the warping act to smear out the difference between the peak positions of the azimuthally averaged radial surface brightness profiles shown in Fig. 8. Fortunately, overlapping should have little effect in the regions located along the major axis of the Cartwheel.

We apply an alternative method for determining the angular difference between the peak positions of radial profiles in different photometric bands. Instead of computing the fluxes from each elliptical annulus shown in Fig. 7, i.e. the fluxes averaged over the whole azimuth, we make a series of radial cuts drawn from the Cartwheel's nucleus with an angular separation of $0.2^{\circ}$. A series of 360 radial surface brightness profiles of the Cartwheel's outer ring were then constructed by azimuthally averaging these cuts over a $1^{\circ}$ sector. Peaks were located by both fitting a Gaussian to each profile and determining the "center of mass" of each profile. The radial positions of each peak were corrected for a $41^{\circ}$ inclination of the Cartwheel and re-calculated for a zero inclination.

Both methods give very similar results. Angular differences in arcsec between the peak positions are shown in Fig. 9 as a function of position angle, which is measured counterclockwise from a line extending north from the outer ring center. The $K s$-band and $B$-band peaks are located at smaller radii than the peak in $\mathrm{H} \alpha$ for almost all position angles. The angular difference between the $K s$-band and $\mathrm{H} \alpha$ peaks is systematically 


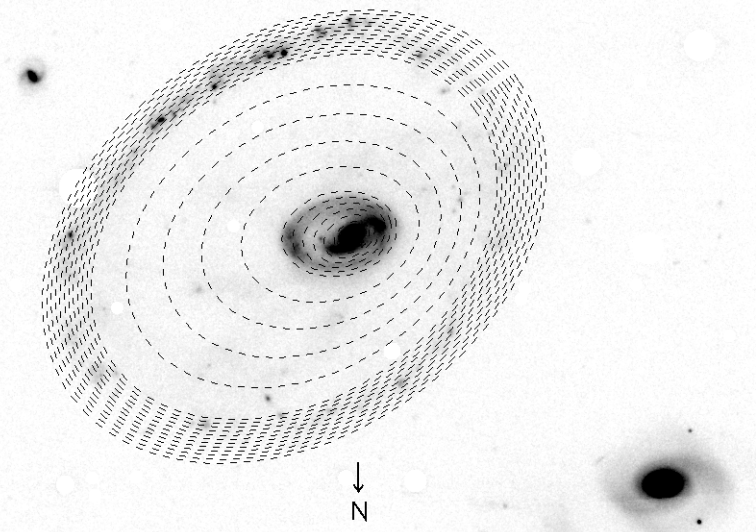

Fig. 7. The Cartwheel galaxy in $K s$-band. The dashed ellipses outline elliptical annuli used for determining the azimuthally averaged radial surface brightnesses of the Cartwheel. The north is shown by the arrow.

bigger than between the $B$-band and $\mathrm{H} \alpha$ peaks (Fig. 9b), which is in full agreement with our model predictions.

Let us now consider the effects of finite thickness of the Cartwheel's disk in more detail. Let us assume that the Cartwheel's disk has a radially non-constant thickness, with the inner regions being thicker than the outer regions. This assumption is justified, since the inner regions of the Cartwheel are expected to be older and hence thicker than the outer regions. Internal dust extinction is known to be considerable in the Cartwheel's outer ring (Fosbury \& Hawarden 1977, VB). We assume a limiting case when part of the Cartwheel's outer ring that is located below the galactic plane is totally unseen. Under these assumptions, the angular differences between the peak positions should have maximums along the minor semiaxis that is drawn on the galaxy's near side. The minima of angular differences are then expected to be along the minor semiaxis drawn on the galaxy's far side. We smoothed Fig. 9 with a $30^{\circ}$ wide median filter in order to minimize the noise. The results are shown by the solid lines in each panel of Fig. 9. The angular differences between all three bands are minimal along the southern minor semi-axis at position angles $180^{\circ}-220^{\circ}$. On the opposite side, along the northern minor semi-axis at position angles $20^{\circ}-40^{\circ}$, the angular differences in Fig. 9 show an expected tendency to grow and reach a maximum. This places the southern part of the Cartwheel on the galaxy's far side. Our conclusion is consistent with the analysis of Higdon (1996), who deduced the galaxy's orientation indirectly from the form of rotation curve and spokes. There is however an unexpected maximum in the $\mathrm{H} \alpha-K$ and $\mathrm{H} \alpha-B$ angular peak differences at position angles $320^{\circ}-340^{\circ}$, while the $B-K$ angular peak difference is minimal there. This may be a manifestation of an $\mathrm{H} \alpha$ warp in the Cartwheel's outer ring.

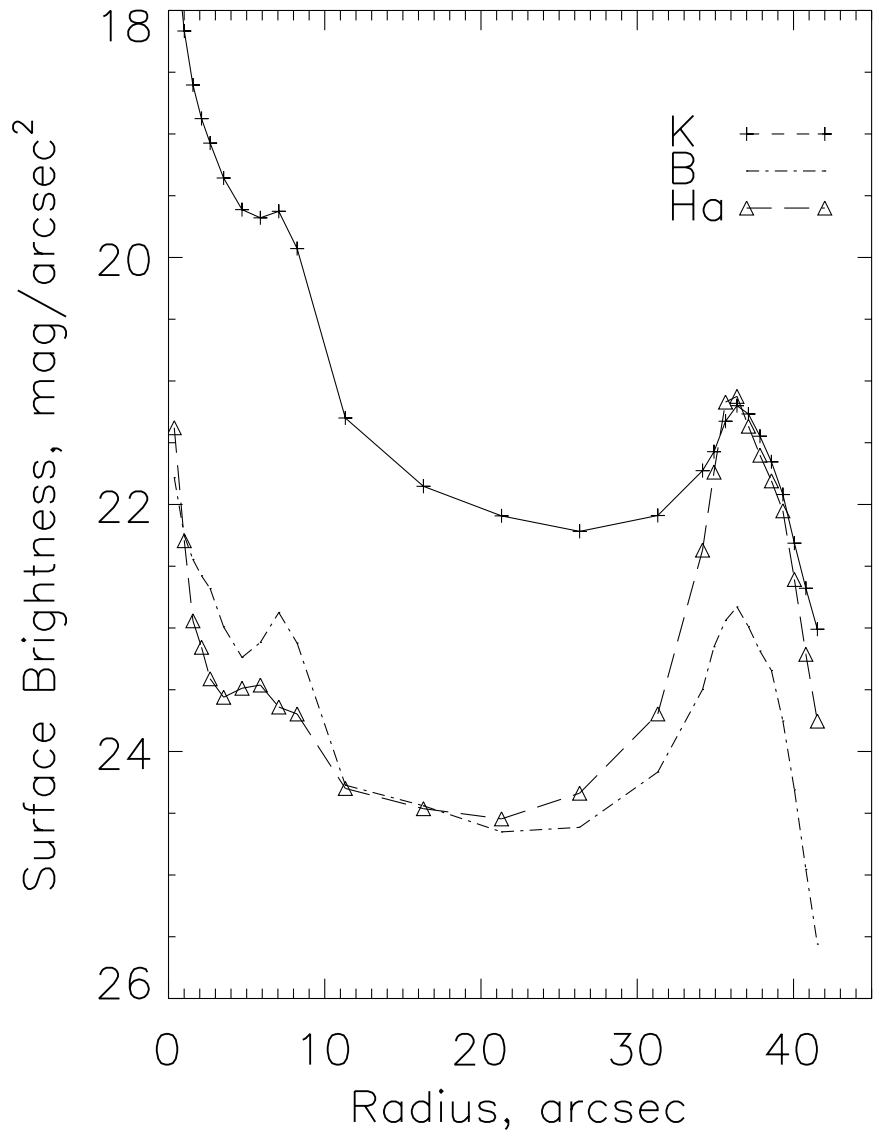

Fig. 8. The azimuthally averaged surface brightness profiles of the Cartwheel galaxy. All profiles peak at the position of the outer ring at $36^{\prime \prime}$. No difference between the peak positions is seen.

As it has been noted above, differences between the peak positions measured along the major axis are not influenced by the finite thickness of a ring galaxy. Hence, they might be considered as an indicator of the Cartwheel's outer ring propagation velocity. The angular peak differences along the Cartwheel's major axis determined from the smoothed curves in Fig. 9 are $\mathrm{H} \alpha-K s=1.97^{\prime \prime}, \mathrm{H} \alpha-B=1.29^{\prime \prime}$, and $B-K s=0.67^{\prime \prime}$. They correspond to the linear peak differences of $\mathrm{H} \alpha-K s=1.3 \mathrm{kpc}, \mathrm{H} \alpha-B=0.85 \mathrm{kpc}$, and $B-K s=0.45 \mathrm{kpc}$ for the adopted distance of $140 \mathrm{Mpc}$. These values are even bigger than those predicted by our modeling for the Cartwheel's outer ring velocity of $90 \mathrm{~km} \mathrm{~s}^{-1}$, implying that the outer ring is currently propagating at $v_{\text {ring }} \approx 110 \mathrm{~km} \mathrm{~s}^{-1}$. We note that these estimates cannot be considered as very accurate, though they clearly show that the Cartwheel's outer ring is indeed expanding, and probably with a considerable velocity as predicted by modeling of color gradients in Sect. 2 and numerical hydrodynamics modeling in Sect. 3 . We derive $v_{\text {ring }} \approx 100 \mathrm{~km} \mathrm{~s}^{-1}$ as an average of estimates obtained in Sect. 2-4. Since the wave velocity most probably decrease with time, we conclude that the Cartwheel galaxy is no older than $250 \mathrm{Myr}$. 

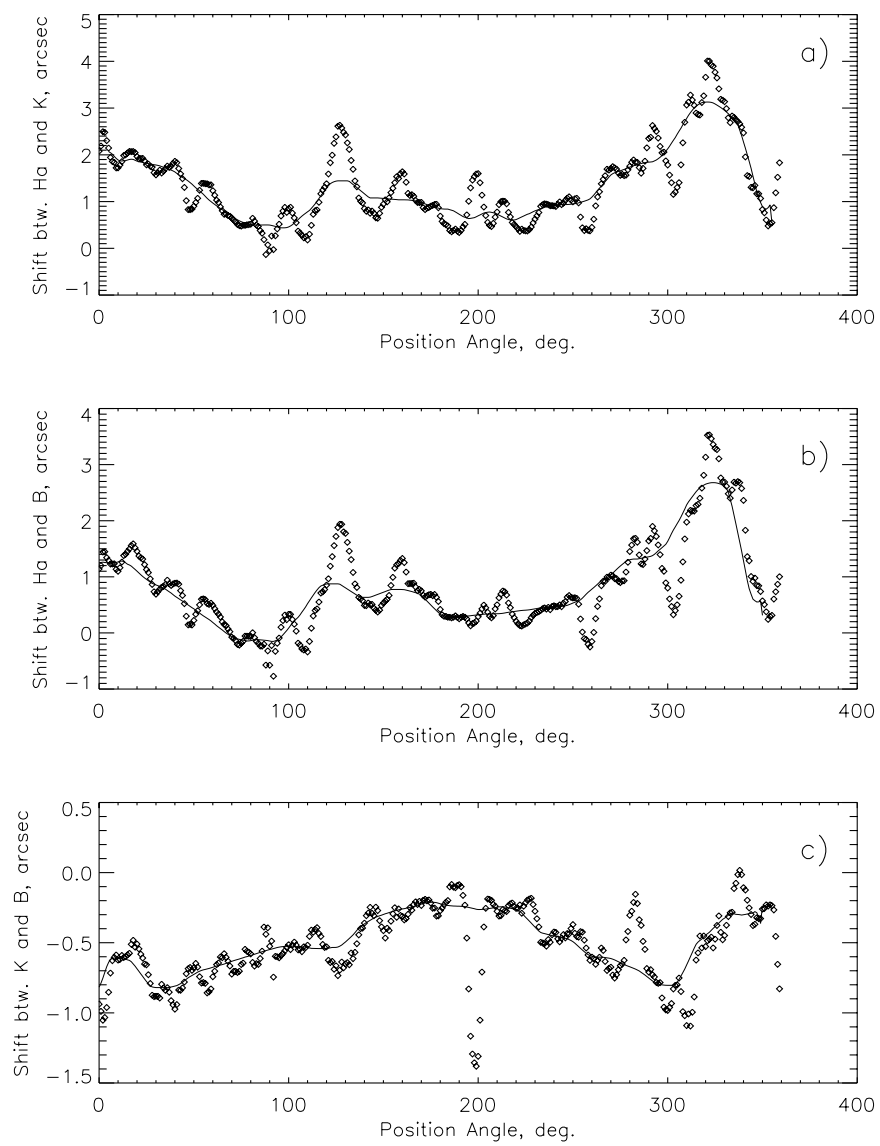

Fig. 9. The angular difference between the peaks in radial surface brightnesses azimuthally averaged over a $1^{\circ}$ sector as a function of position angle measured counterclockwise from a line extending north from the outer ring center. a) Angular difference between the $\mathrm{H} \alpha$ and $K s$-band peaks, b) angular difference between the $\mathrm{H} \alpha$ and $B$-band peaks, c) angular difference between the $K s$ - and $B$-band peaks. Solid lines indicate the angular peak differences smoothed by a wide median filter $\left(30^{\circ}\right)$.

\section{Other possibilities for estimating the propagation velocity of ring gas density waves}

\subsection{Call triplet equivalent widths}

The CaII triplet $(\mathrm{CaT})$ lines originate in the atmospheres of cool stars with the absorption being the strongest in red supergiants. While studying numerically the evolutionary behavior of the CaT equivalent widths $(\mathrm{EW}(\mathrm{CaT}))$ in starbursts of different metallicities, Mayya (1997) found that besides the primary peak around $10 \mathrm{Myr}$ the $\mathrm{EW}(\mathrm{CaT})$ show a well defined secondary peak around $60 \mathrm{Myr}$ in the solar metallicity starbursts. The time separation $t_{\mathrm{CaT}}=50 \mathrm{Myr}$ between the primary $\mathrm{EW}(\mathrm{CaT})$ peak around $10 \mathrm{Myr}$ and the secondary $\mathrm{EW}(\mathrm{CaT})$ peak around $60 \mathrm{Myr}$ might be in principle used to infer the propagation velocity of gas density waves in ring galaxies.

We illustrate this possibility for the Cartwheel ring galaxy. We assume that the Cartwheel's outer ring is currently propagating at $v_{\text {ring }}=90 \mathrm{~km} \mathrm{~s}^{-1}$. For the time being, we assume that the Cartwheel's gaseous disk has the solar metallicity. We also neglect the possible input to $\mathrm{EW}(\mathrm{CaT})$ from the pre-collision stellar populations, which is indeed minimal at the Cartwheel's outer parts (VB). The filled triangles in Fig. 10 show the model radial profile of $\mathrm{EW}(\mathrm{CaT})$ after the outer ring has propagated $24 \mathrm{kpc}$ in the galactic disk. Two well-defined peaks of $\mathrm{EW}(\mathrm{CaT})$ at $19.1 \mathrm{kpc}$ and $23.5 \mathrm{kpc}$ are clearly evident. For the outer ring propagation velocity of $90 \mathrm{~km} \mathrm{~s}^{-1}$ it takes exactly $50 \mathrm{Myr}$ to cross the distance between these two peaks in Fig. 10. The angular difference between the peak positions of $6.6^{\prime \prime}$ should be easily detected. Now given the linear difference between the primary and secondary peak positions $R_{\text {CaT }}$ is known from observations of a ring galaxy, one can calculate the propagation velocity of gas density wave by dividing $R_{\mathrm{CaT}}$ on the value of $t_{\mathrm{CaT}}$. Or alternatively, if propagation velocity is known from independent measurements or/and detailed modeling, one can calculate $R_{\mathrm{CaT}}$, thus breaking new ground for the distance-independent estimates of a ring galaxy linear diameter. Hence, EW(CaT) may provide the means for a Hubble-constant-independent estimate of a distance to a ring galaxy.

This method of evaluating the propagation velocity of gas density waves is most effective in the solar metallicity environments. For sub-solar metallicities however the primary and secondary peaks in $\mathrm{EW}(\mathrm{CaT})$ become much less pronounced as shown by the open squares in Fig. 10 for the $z_{\odot} / 2.5$ metallicity. In fact, the primary peak almost disappear for metallicities below $z_{\odot} / 5$. Furthermore, the absolute values of $\mathrm{EW}(\mathrm{CaT})$ generally decrease for progressively lower metallicities (Mayya 1997). These difficulties make the observational detection of the primary and secondary peaks problematic in the sub-solar metallicity environments. For the Cartwheel galaxy, the solar metallicity is expected only in the central parts. The outer parts are most probably of sub-solar $\left(z_{\odot} / 3.5\right)$ metallicity and this method will not work. Unfortunately, heavy element abundance measurements of Bransford et al. (1998) indicate that individual star-forming knots in the rings of all ring galaxies in their sample have sub-solar metallicities in the range from $z_{\odot} / 2.5$ to $z_{\odot} / 5$. We have to look for metal-rich ring galaxies in order to apply this method for determining the propagation velocity of gas density wave.

\subsection{Other spectral lines}

Some other prominent absorption lines might be used for estimating the propagation velocity of gas density waves in ring galaxies. Synthesized spectra of a single-burst stellar population (PEGASE2 or Starburst99) indicate that the equivalent widths of such optical absorption lines as $\mathrm{Mg} \mathrm{b}, \mathrm{Na} \mathrm{D}$, and some of strong Fe lines have a local maximum/minimum between 10 and $250 \mathrm{Myr}$. A set of these lines can help to establish "time labels" along the radius of a ring galaxy under an assumption that most stars in the galaxy were born in a single star formation event. Even two localizations of such extremes in the radial profiles of absorption line equivalent widths can provide us with the ages of stellar populations at different radii and hence give us an insight into the value of propagation velocity of gas density wave that triggered the star formation event. Use of a large aperture telescope in combination with a 


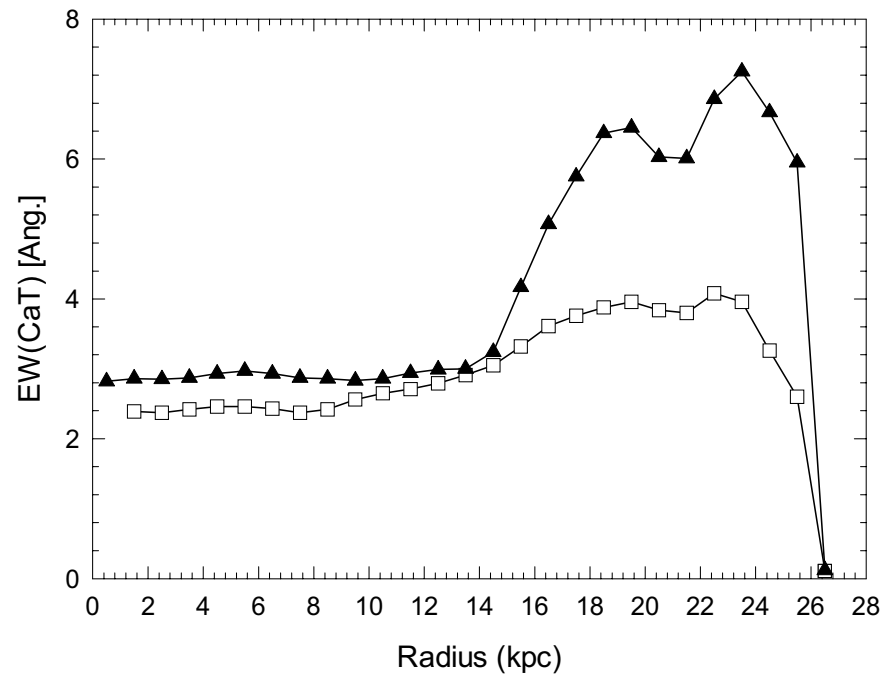

Fig. 10. The model radial profile of $\mathrm{EW}(\mathrm{CaT})$, as would be expected if the Cartwheel's disk had the solar metallicity (filled triangles) and $z_{\odot} / 2.5$ metallicity (open squares).

contemporary spectrograph makes it possible to obtain high signal-to-noise spectra of ring galaxies in a few hours of integration time (Moiseev 2002).

\subsection{Non-thermal radio emission}

Evolved stellar populations in the wake of an expanding ring density wave explode as supernovae. Type II supernova remnants are responsible for strong non-thermal radio emission. Condon and Yin (1990) argue that most Type II supernova remnants and their consequent non-thermal radio emission result from stars with masses just over $8 M_{\odot}$, while stars more massive than $20 M_{\odot}$ account for most of the ionizing radiation and consequent $\mathrm{H} \alpha$ emission. If so, we might expect to observe the difference between the peak positions of the radial profiles of non-thermal radio emission and $\mathrm{H} \alpha$ surface brightness in ring galaxies. Indeed, the ring galaxy VII Zw 466 does show signs of such a difference when radio emission peaks on the inside edge of the disk and does not coincide with the prominent HII region complexes. The radial difference between the peaks in $\mathrm{H} \alpha$ and non-thermal radio emission might be used to infer the propagation velocity of gas density waves in ring galaxies in the same manner as in Sect. 4.

\section{Conclusions}

Our numerical hydrodynamics modeling of ring galaxy formation demonstrates that the maximum radial expansion velocity of gas $\left(v_{\text {gas }}\right)$ in the first ring is always below the propagation velocity of the first ring itself $\left(v_{\text {ring }}\right)$ The inequality $v_{\text {gas }}<v_{\text {ring }}$ is less strict in ring galaxies of comparable disk/halo masses than in halo-dominated ring galaxies. Numerical hydrodynamics modeling of the Cartwheel galaxy indicates the outer ring is currently propagating at $v_{\text {ring }} \approx 100 \mathrm{~km} \mathrm{~s}^{-1}$, while the maximum radial expansion velocity of gas in the outer ring is currently $v_{\text {gas }} \approx 65 \mathrm{~km} \mathrm{~s}^{-1}$. The latter value is in marginal agreement with the measurements of Higdon (1996) based on HI kinematics, but substantially disagree with the measurements of Amram et al. (1998) based on kinematics of ionized gas. We conclude that the dynamical properties of neutral and ionized gas may differ significantly in ring galaxies.

We demonstrate that the radial $B-V / V-K$ color gradients, together with the $Q_{B V K}$ extinction-free indices, can be used for estimating the value of $v_{\text {ring }}$ in ring galaxies. Modeling of the radial $B-V / V-K$ color gradients measured in the Cartwheel disk by Marcum et al. (1992) is sensitive to the assumed value of a Hubble constant. Nevertheless, the color gradient modeling imposes the lower limit of $v_{\text {ring }}=40 \mathrm{~km} \mathrm{~s}^{-1}$ on the propagation velocity of the outer ring for any value of a Hubble constant in the range $H_{0}=[50-100] \mathrm{km} \mathrm{s}^{-1} \mathrm{Mpc}^{-1}$. For the adopted in this work $H_{0}=65 \mathrm{~km} \mathrm{~s}^{-1} \mathrm{Mpc}^{-1}$, the best agreement between the model and observed radial color gradients is achieved for velocities of $90 \mathrm{~km} \mathrm{~s}^{-1}$ and even higher.

Our modeling of the Cartwheel galaxy demonstrates that the angular differences between the peaks in the $\mathrm{H} \alpha, K$-, and $B$ band radial surface brightness profiles are $\mathrm{H} \alpha-K=1.65^{\prime \prime}$ and $\mathrm{H} \alpha-B=1.05^{\prime \prime}$, which should be detected with the achieved resolution. On the other hand, we find that the azimuthally averaged radial $\mathrm{H} \alpha$ surface brightness profile of the Cartwheel's outer ring does not peak exterior to those in $K s$ - and $B$-bands, contrary to the predictions of our model for $v_{\text {ring }}=90 \mathrm{~km} \mathrm{~s}^{-1}$. We conclude that a $41^{\circ}$ inclination, along with a finite thickness and warping of the Cartwheel's disk, act to smear out the angular differences between the peaks in the $\mathrm{H} \alpha, K s$, and $B$ radial profiles. Indeed, we construct a series of the radial surface brightness profiles azimuthally averaged over a $1^{\circ}$ sector. These pie-chart-like radial profiles do show an expected tendency and the $\mathrm{H} \alpha$ radial profile peaks exterior to those in $K s$ and $B$-bands at the position of the Cartwheel's outer ring. The $\mathrm{H} \alpha-K s$ and $\mathrm{H} \alpha-B$ peak differences are the biggest along the Cartwheel's major axis, where effects of inclination and finite thickness are minimized, and are in close agreement with our model predictions. The peak differences obtained along the Cartwheel's major axis imply $v_{\text {ring }} \approx 110 \mathrm{~km} \mathrm{~s}^{-1}$. Azimuthal variations in $\mathrm{H} \alpha-K s, \mathrm{H} \alpha-B$, and $B-K s$ peak differences support our assumptions about a thick and non-flat nature of the Cartwheel's outer ring. We conclude that the Cartwheel's outer ring is currently propagating at $v_{\text {ring }} \approx 100 \mathrm{~km} \mathrm{~s}^{-1}$, which is in agreement with our numerical hydrodynamics results.

We show that the equivalent widths of some spectral lines, such as the CaII triplet, might be used for determining the propagation velocity of ring density waves in solar metallicity environments. Solar metallicity rings have not been detected so far (Bransford et al. 1998), but they may be expected in young ring galaxies where the rings have not yet expanded out to the metal-unpolluted regions. Non-thermal radio emission is also a promising tool for determining the wave velocity in ring galaxies with high rates of star formation like the Cartwheel.

Acknowledgements. We are thankful to the referee, Dr. A.P. Marston, for his suggestions and critical comments, which helped us to improve the paper. The authors are grateful to Dr. Y.D. Mayya for providing his population synthesis code. We thank Dr. S.Popov for help with data collection. The paper is based on observations made with ESO Telescopes at the Paranal Observatory under program ID 66.B0666(B). Some of the data presented in this paper were obtained from 
the Multimission Archive at the Space Telescope Science Institute (MAST). STScI is operated by the Association of Universities for Research in Astronomy, Inc., under NASA contract NAS5-26555. Support for MAST for non-HST data is provided by the NASA Office of Space Science via grant NAG5-7584 and by other grants and contracts. The paper is based on archival data taken from the Canadian Astronomy Data Center, which is operated by the Dominion Astrophysical Observatory for the National Research Council of Canada's Herzberg Institute of Astrophysics. Partial support from RFBR 00-02-17689 is acknowledged.

\section{References}

Amram, O., Mendes de Oliveira, C., Boulesteix, J., \& Balkowaki, C. 1998, A\&A, 330, 881

Appleton, P. N., \& Higdon, J. L. 1993, ApJ, 411, 108

Appleton, P. N., \& Marston, A. P. 1997, AJ, 113, 201

Appleton, P. N., \& Struck-Marcell, C. 1987, ApJ, 318, 103

Athanassoula, E., Puerari, I., \& Bosma, A. 1997, MNRAS, 286, 284

Bahcall, J. 1984, ApJ, 276, 156

Binney, J., \& Merrifield, M. 1998, in Galactic Astronomy, ed. P. Ostriker, \& D. N. Spergel (Princeton University Press), 442

Bohlin, R. C., Savage, B. D., \& Drake, J. F. 1978, ApJ, 224, 225

Bransford, M. A., Appleton, P. N., Marston, A. P., \& Charmandaris, V. 1998, AJ, 116, 2757

Charlot, S. 1996, in From Stars to Galaxies, ed. C. Leitherer, U. Fritze-v. Alvensleben, \& J. Huchra (San Francisco: ASP), ASP Conf. Ser., 98, 275

Combes, F., \& Gerin, M. 1985, A\&A, 150, 325

Condon, J. J., \& Yin, Q. F. 1990, ApJ, 357, 97

Davies, R. L., \& Morton, D. C. 1982, MNRAS, 201, 69

Faber, S. M., \& Gallagher, J. S. 1979, ARA\&A, 17, 135
Fioc, M., \& Rocca-Volmerange, B. 1999 [astro-ph/9912179] Fosbury, R. A. E., \& Hawarden, T. G. 1977, MNRAS, 178, 473 Hernquist, L., \& Weil, M. L. 1993, MNRAS, 261, 804

Higdon, J. L. 1995, ApJ, 455, 524

Higdon, J. L. 1996, ApJ, 467, 241

Horellou, C., Casoli, F., Combes, F., et al. 1995, A\&A, 298, 743

Horellou, G., Charmandaris, V., Combes, F., et al. 1998, A\&A, 340, L51

Korchagin, V., Mayya, Y. D., Vorobyov, E. I., \& Kembhavi, A. K. 1998, ApJ, 495, 757

Korchagin, V., Mayya, Y. D., \& Vorobyov, E. I. 2001, ApJ, 554, 281

Kurucz, R. L. 1992, Stellar Populations of Galaxies, ed. B. A. Renzini (New York: Kluwer), IAU Symp., 149, 225

Leitherer, C., Schaerer, D., Goldader, J. D., et al. 1999, ApJS, 123, 3

Lejeune, T., Buser, R., \& Cuisinier, F. 1997, A\&AS, 125, 229

Marcum, P. M., Appleton, P. N., \& Higdon, J. L. 1992, ApJ, 399, 57

Marston, A. P., \& Appleton, P. N. 1995, ApJ, 109, 1002

Martin, C., \& Kennicutt, R. 2001, ApJ, 555, 301

Mayya, Y. D. 1995, AJ, 109, 2503

Mayya, Y. D. 1997, ApJ, 482, L149

Mihos, J. C., \& Hernquist, L. 1994, ApJ, 437, 611

Moiseev, A. 2002 [astro-ph/0211104], private communication

Olson, K. M., \& Kwan, J. 1990, ApJ, 349, 480

Reshetnikov, V., \& Combes, F. 1997, A\&A, 324, 80

Schaller, G., Schaerer, D., Meynet, G., \& Maeder, A. 1992, A\&AS, 96, 269

Stone, J. M., \& Norman, M. L. 1992, ApJS, 80, 753

Struck, C. 1997, ApJS, 113, 269

Toomre, A. 1978, in The Large-Scale Structure of the Universe, ed. M. S. Longair, \& J. Einasto (D. Reidel Publications, Holland), IAU Symp., 79, 109

Tsuchiya, T., Korchagin, V., \& Wada, K. 1998, ApJ, 505, 607

Vorobyov, E. I., \& Bizyaev, D. 2001, A\&A, 377, 835 (VB) 